\title{
Mitochondrial Acetylation and Diseases of Aging
}

\author{
Gregory R. Wagner' ${ }^{1}$ and R. Mark Payne ${ }^{1,2}$ \\ ${ }^{1}$ Department of Medical \& Molecular Genetics, Riley Heart Research Center, Wells Center for Pediatric Research, \\ Indiana University School of Medicine, Indianapolis, IN 46202, USA \\ ${ }^{2}$ Department of Pediatrics, Riley Heart Research Center, Wells Center for Pediatric Research, \\ Indiana University School of Medicine, IN 46202, USA
}

Correspondence should be addressed to R. Mark Payne, rpayne@iupui.edu

Received 22 October 2010; Accepted 8 January 2011

Academic Editor: Alberto Sanz

Copyright ( 2011 G. R. Wagner and R. M. Payne. This is an open access article distributed under the Creative Commons Attribution License, which permits unrestricted use, distribution, and reproduction in any medium, provided the original work is properly cited.

\begin{abstract}
In recent years, protein lysine acetylation has emerged as a prominent and conserved regulatory posttranslational modification that is abundant on numerous enzymes involved in the processes of intermediary metabolism. Well-characterized mitochondrial processes of carbon utilization are enriched in acetyl-lysine modifications. Although seminal discoveries have been made in the basic biology of mitochondrial acetylation, an understanding of how acetylation states influence enzyme function and metabolic reprogramming during pathological states remains largely unknown. This paper will examine our current understanding of eukaryotic acetate metabolism and present recent findings in the field of mitochondrial acetylation biology. The implications of mitochondrial acetylation for the aging process will be discussed, as well as its potential implications for the unique and localized metabolic states that occur during the aging-associated conditions of heart failure and cancer growth.
\end{abstract}

\section{Introduction}

Over millions of years, eukaryotic organisms evolved finetuned metabolic mechanisms for orchestrating the conversion of diverse carbon substrates into cellular energy. These mechanisms include changes in gene transcription, allosteric regulation of metabolic enzymes by fluxing pools of intermediary metabolites, and direct regulation by posttranslational modifications (PTMs), such as phosphorylation. One PTM, lysine acetylation, has been traditionally studied in the context of nuclear histone modifications and is well known to influence changes in gene expression [1]. However, recent proteomics surveys have revealed that lysine acetylation is a widespread cellular modification that is particularly abundant on mitochondrial proteins, suggesting a new posttranslational mechanism for coordinating the metabolism of carbon sources [2,3]. Accordingly, several studies have demonstrated that reversible lysine acetylation can, by targeting key enzymes, modulate the activity of mitochondria-localized fatty acid $\beta$-oxidation, the tricarboxylic acid cycle (TCA), urea cycle, and oxidative phosphorylation in a nutrientresponsive manner [4-7]. The reversible and nutrientsensitive manner of many of these acetyl modifications has strongly implicated the mitochondrial-localized members of the $\mathrm{NAD}^{+}$-dependent deacetylases (sirtuins) as regulatory mediators of these fundamental metabolic processes [8]. Sirtuins are also believed to be part of a genetic program influencing the development of age-related conditions such as heart disease, neurodegenerative disease, and cancer [9]. Although seminal discoveries have been made in the basic biology of mitochondrial acetylation, an understanding of how acetylation states influence enzyme function and metabolic reprogramming during age-associated pathological states remains largely unknown.

In this paper we will review mammalian acetate metabolism and provide a brief history of lysine acetylation as a means to discussing more recent advances in mitochondrial acetylation biology. We then suggest a role for alterations in mitochondrial acetylation states during agerelated conditions of heart failure and cancer, as well as review its potential role in human longevity.

\section{Acetyl-CoA and Metabolism}

Acetate is an ancient energy precursor molecule of metazoan metabolism (Figure 1). In eukaryotic systems, the principal 
source of cellular acetate is generated by the mitochondrial processes of glucose-derived pyruvate oxidation, amino acid catabolism, and the oxidation of even-numbered fatty acyl chains. These distinct metabolic pathways are all capable of yielding the activated form of cellular acetate, or acetyl-CoA. Thus, acetyl-CoA represents a common convergence point for carbohydrate, amino acid, and lipid catabolism. How mitochondrial acetyl-CoA is utilized throughout the cell is heavily dependent on the cell type and the metabolic state of the organism. However, the primary function of acetylCoA throughout all cell types is as a carbon donor in the tricarboxylic acid (TCA) cycle. Originally characterized by Hans Krebs and Albert Szent-Györgyi, the TCA cycle is a series of substrate oxidation and decarboxylation reactions that serve to liberate carbon dioxide and generate electron donors in the form of the reduced coenzymes nicotinamide adenine dinucleotide (NADH) and flavin adenine dinucleotide $\left(\mathrm{FADH}_{2}\right)$ [10]. The coenzymes $\mathrm{NADH}$ and $\mathrm{FADH}_{2}$, in turn, serve as high-energy electron donors which are oxidized by the respiratory chain complexes to generate the proton-motive electrochemical gradient that is ultimately responsible the formation of energy-rich ATP [11]. AcetylCoA also serves as a vital component of anabolic cellular processes. In general, during a state of nutrient excess (fed state), a proportion of the excess acetyl-CoA generated within the mitochondria is exported into the cytosol in the form of citrate, where it is reconverted into acetylCoA by ATP-citrate lyase [12]. This cytosolic acetyl-CoA can then participate in de novo synthesis of fatty acids and sterols that are vitally important for the formation and maintenance of lipid membranes, as well as the synthesis of steroid hormones, triacylglycerols, cholesterol, and fatsoluble vitamins $[13,14]$. Lipogenesis primarily occurs in hepatocytes and adipocytes, whereas cholesterol synthesis primarily occurs in hepatocytes. In contrast to the fed state, during a nutrient-depleted state, the liver uses TCA cycle intermediates for gluconeogenesis which depletes oxaloacetate and limits the entry of acetyl-CoA into the TCA cycle. As the liver continues to oxidize fatty acids for fuel, acetylCoA begins to accumulate and is converted into the transport forms of acetate known as ketone bodies. Ketone bodies are then used as an alternative fuel source in the brain, heart, and skeletal muscle during fasting or glucose-scarce conditions [15]. Free cytoplasmic and mitochondrial acetate can also be converted into acetyl-CoA via the ATP-dependent mechanisms of acetyl-CoA synthetase 1 and 2, respectively $[16,17]$. Interestingly, acetyl-CoA synthetase 2 (AceCS2) was recently found to be an enzyme critically involved in thermogenesis during fasting conditions [18]. Through its well-established roles in the generation of cellular energy and as a macromolecular building block, acetyl-CoA stands as a critical chemical intermediate that is inextricably linked to cellular energy homeostasis. However, the fundamental and ancient link between acetyl-CoA, mitochondria, and metabolism is underappreciated in the context of acetylCoA's exciting and dynamic use as the acetyl group donor for acetyl-lysine posttranslational modifications. Interestingly, a recent study highlighted this often overlooked aspect of acetyl-CoA metabolism by demonstrating that the metabolic enzyme ATP-citrate lyase, in addition to its previously mentioned roles in lipid and steroid synthesis, also regulates global patterns of histone acetylation and corresponding changes in gene expression [19]. Therefore, it is nutrient availability and metabolism that largely determine the cellular pool of acetyl-CoA available for acetyl-lysine protein modifications.

\section{3. $\mathbf{N}^{\varepsilon}$-Acetylation}

Acetylation occurring on the epsilon-amino group of lysine residues $\left(\mathrm{N}^{\varepsilon}\right.$-acetylation) was discovered on histone proteins purified from calf thymus over forty years ago [20]. The ensuing studies of $\mathrm{N}^{\varepsilon}$-acetylation focused almost exclusively on histone substrates [21-23]. It was not until 1996 that Taunton et al. purified the first histone deacetylase enzyme [24]. This important discovery quickly expanded research interest in the field of histone acetylation and in the past 15 years has led to the discovery of numerous enzymes that catalyze the addition and removal of acetyl groups, termed histone acetyltransferases (HATs) and histone deacetylases (HDACs), respectively. The dynamic and opposing functions of HATs and HDACs form the basis for the transcriptional modulation of chromatin and the histone code hypothesis $[1,25]$. Due to their role in global cellular processes of transcription, HDACs have become attractive chemotherapeutic targets. Vorinostat (suberoylanilide hydroxamic acid) and Romidepsin (FK228) are FDA-approved HDAC inhibitors that are currently being used for the treatment of cutaneous T-cell lymphomas, and there are a number of related compounds in clinical trials for other neoplastic indications [26].

Owing to the highly conserved nature of HATs and HDACs across multiple eukaryotic species, we commonly classify them according to their homologs in yeast [27]. There are three primary families of HATs. These include the MYST family, the Gcn-5-related N-acetyltransferases (GNATs), and the E1A-associated protein of $300 \mathrm{kDa} / \mathrm{CREB}-$ binding protein (p300/CBP) family [28]. HDACs are broadly classified into the Rpd3/Hda1 family of deacetylases, and the silent information regulator-2-(Sir2-) like NAD ${ }^{+}$-dependent deacetylases/mono-ADP-ribosyltransferases, or sirtuins. The Rpd3/Hda1 family are further grouped into classes I, II, and IV, while the sirtuins represent class III deacetylases [9, 29]. Due to the histone-centric nature of the founding studies on acetylation, these acetylase and deacetylase enzymes are traditionally referred to as HATs and HDACs. However, research in the past 10 years has established that the socalled HATs and HDACs have multiple nonhistone and extranuclear targets including, among many others, the tumor suppressor $\mathrm{p} 53$, the oncogene $\beta$-catenin, and the molecular chaperone HSP90 [30-33]. Accordingly, these enzymes are now more generally referred to as lysine acetyltransferases (KATs) and lysine deacetylases (KDACs).

Biochemically, the positively charged $\varepsilon$-amino group of lysine residues is important for protein stability via hydrogen bonding with nearby amino acid residues and can also serve as a base during enzyme catalysis [34] (Figure 2). The covalent enzymatic addition of an acetyl moiety to the $\mathcal{\varepsilon}$-amino 


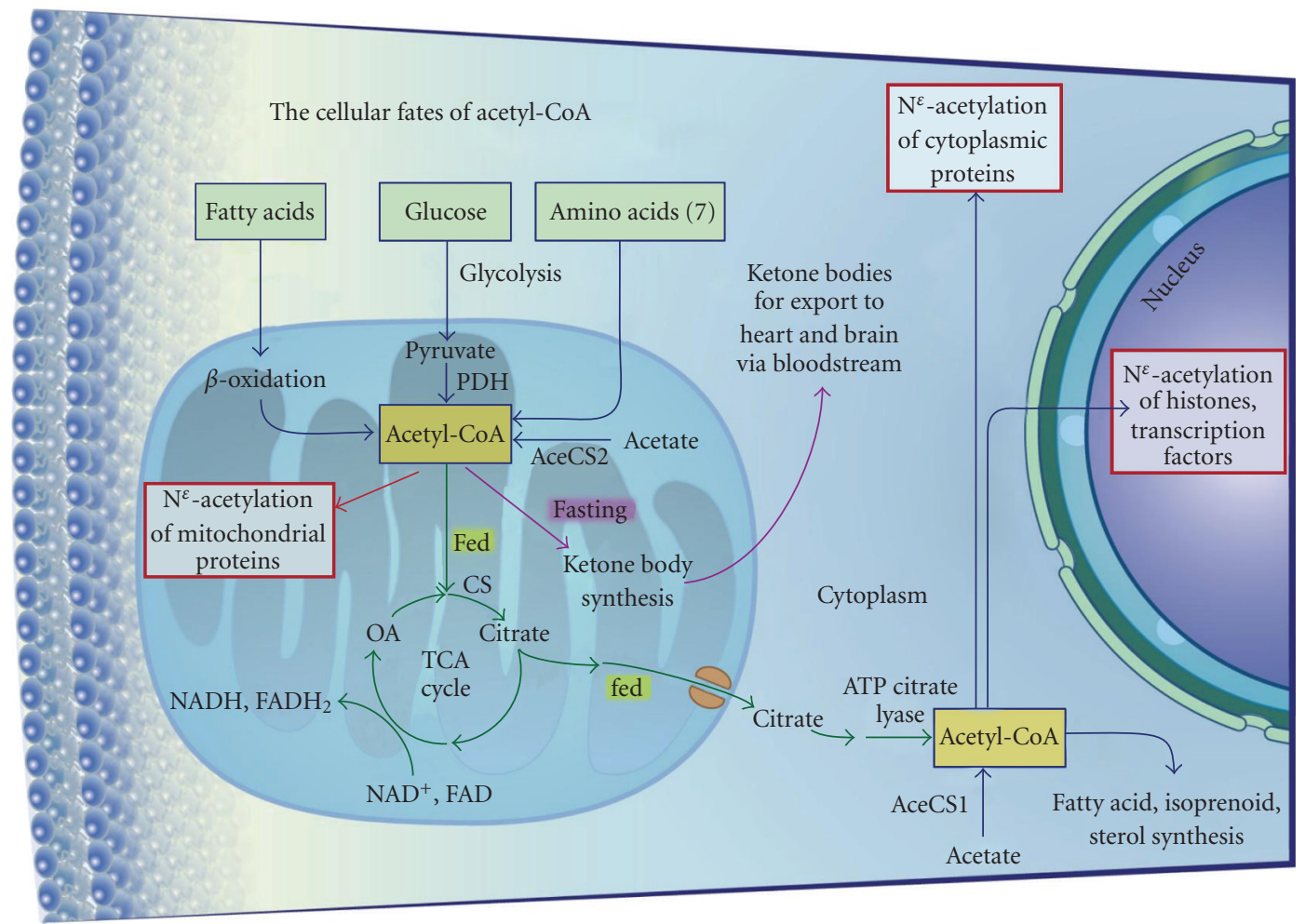

Figure 1: The cellular fates of acetyl-CoA. Please see section entitled "Acetyl-CoA and Metabolism" for a detailed description of the figure. PDH: pyruvate dehydrogenase complex, AceCS2: acetyl-CoA synthetase 2, CS: citrate synthase, OA: oxaloacetate, AceCS1: acetyl-CoA synthetase 1.

group on a lysine residue effectively neutralizes its native positive charge, which changes its propensity to interact with nearby residues, other proteins, or can alter the activity of an enzyme. In its well-established chromatin context, acetylation neutralizes positively charged lysine residues on histone tails, thereby reducing their charged interactions with chromatin and providing an epigenetic mark which is recognized and used by bromodomain-containing proteins to recruit transcriptional machinery and other chromatinmodifying elements [1]. In another context, acetylation of lysine 88 on the enzyme ornithine transcarbamylase (OTC) changes its affinity for its substrate, carbamoyl phosphate, and negatively regulates the detoxification of ammonia in the urea cycle [35]. Additionally, acetylation of lysine 685 on STAT3 promotes its stable dimerization and subsequent function as a transcriptional activator [36]. The $\varepsilon$-amino groups of lysine residues are also the targets of a number of other posttranslational modifications including methylation, ubiquitination, SUMOylation, and NEDDylation. These other modifications may confer their own unique properties to proteins and protein functions that could be coordinated with acetylation/deacetylation. Ubiquitination of lysine residues, in particular, is known to cooperate with acetylation to regulate the proteolytic degradation of cellular proteins [37]. It has also been proposed that acetylation may also act in concert with other distinct posttranslational modifications to achieve a broad spectrum of functional protein outcomes that has been termed the "protein modification code," analogous to the "histone code" for transcriptional regulation [38]. In general, it has become clear in recent years that $\mathrm{N}^{\varepsilon}$-acetylation is a widespread and functionally diverse posttranslational modification.

\section{4. $\mathrm{NAD}^{+}$Dependent Deacetylases (Sirtuins)}

In their traditional nuclear context, KATs catalyze the acetylCoA-dependent addition of acetyl groups to disordered histone tails - a mark that generally induces a "loose" and transcriptionally active conformation of chromatin. In contrast, the traditional functional understanding of KDACs holds that they catalyze the $\mathrm{H}_{2} \mathrm{O}$-dependent removal of acetyl groups from histone tails to induce "tight" and transcriptionally repressed chromatin [25]. This well-established paradigm for transcriptional modulation was broadened with the discovery of the mechanistically distinct sirtuin class of deacetylases/mono-ADP ribosyltransferases.

There are 7 mammalian sirtuin enzymes. The nuclearlocalized family members consist of SIRT1, SIRT6, and SIRT7, while SIRT2 is concentrated in the cytosol [9]. Interestingly, SIRT3, SIRT4, and SIRT5 exhibit mitochondrial localization $[9,39,40]$. A recent report has also suggested that SIRT1 could function within the mitochondria [41]. Together, the sirtuin family of KDACs are implicated in the regulation of a broad scope of biological processes including, but not limited to, transcriptional repression, development, apoptosis, DNA repair, cellular stress responses, and 

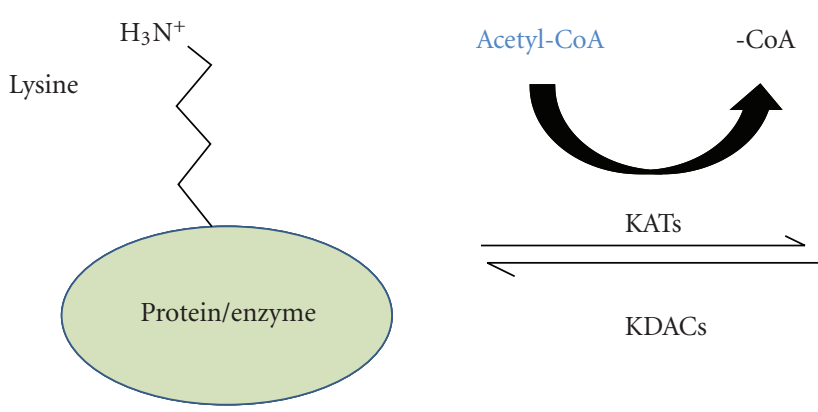

KDACs

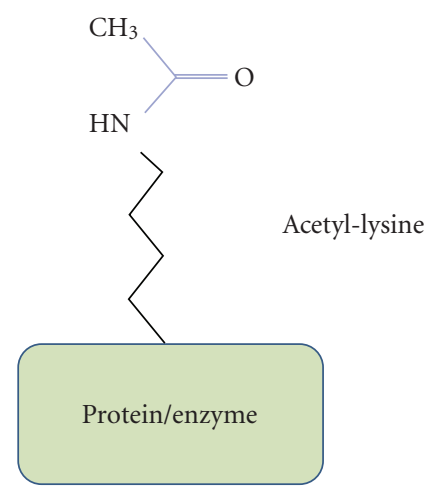

(i) Positively charged interactions and hydrogen bonding with nearby elements

(ii) Free catalytic base

(iii) Promotes heterochromatin formation on histones

(iv) Can be ubiquitinated (less stable ?)

(v) Active and inactive metabolic enzymes (i) Neutral charge and different hydrogen bonding profile

(ii) Lacks catalytic base

(iii) Promotes euchromatin formation on histones

(iv) Cannot be ubiquitinated (more stable ?)

(v) Active and inactive metabolic enzymes

(vi) Crosstalk with other PTMs ?

(phosphorylation, SUMOylation, etc.)

FIGURE 2: Changes in protein function and stability known to be associated with lysine acetylation. KATs: lysine acetyltransferases, KDACs: lysine deacetylases.

metabolism [42-46]. Sirtuins are unique among the four families of mammalian KDACs in that they catalyze the removal of acetyl groups in a fashion that is dependent on the cellular coenzyme $\mathrm{NAD}^{+}$. The reduced form of $\mathrm{NAD}^{+}$, $\mathrm{NADH}$, is generally produced during cellular catabolic processes that serve to derive energy from nutrients, whereas the oxidized form, $\mathrm{NAD}^{+}$, accumulates within cells during nutrient scarce conditions. Thus, the ratio of the reduced form to the oxidized form, or $\mathrm{NAD}^{+} / \mathrm{NADH}$, is indicative of the overall cellular energy state. Sirtuin deacetylase activity is responsive to this measure of cellular energetic balance and requires $\mathrm{NAD}^{+}$as the acceptor of an acetyl group from a target acetyl-lysine residue or for protein ADP ribosylation [47]. The $\mathrm{NAD}^{+}$-dependent deacetylase reaction yields a deacetylated target protein, $2^{\prime}$-O-acetyl-ADP-ribose, and nicotinamide, which can then act to inhibit the sirtuin reaction mechanism [48]. In general, when the cellular $\mathrm{NAD}^{+} / \mathrm{NADH}$ ratio is elevated during times of exercise, nutrient restriction, or outright fasting, sirtuins are presumed to be more actively deacetylating their target proteins, whereas during a fed or basal state, NADH predominates, and sirtuins are less active. It is via their nutrient and stress-sensing capacity to modify transcriptional landscapes and modulate enzyme function that sirtuins are believed to mediate the healthful benefits of caloric restrictionthe only natural means of consistently extending lifespan and delaying the onset of age-related pathologies such as cancer in mammalian systems $[8,49-52]$. It is the recently understood and growing influence of the sirtuin family on the biological mechanisms of aging that has excited the research community and has made the sirtuins highly attractive therapeutic targets for diseases of aging [53].

\section{Mitochondrial Sirtuins and $\mathbf{N}^{\varepsilon}$-Acetylation}

Mitochondria are key organelles for intermediary metabolism as they coordinate the conversion of cellular carbon sources into useable energy in the form of adenosine triphosphate (ATP). The metabolic processes culminating in ATP synthesis are well characterized and are regulated by various mechanisms including nutrient availability, phosphorylation, allosteric mechanisms, reactive oxygen species, and divalent cations such as calcium and magnesium [5457]. In light of the established understanding that mitochondria are the primary cellular generators of the acetylCoA necessary for enzymatic acetylation, it is surprising that $\mathrm{N}^{\varepsilon}$-acetylation within the mitochondria remained virtually unstudied until the last decade. The discovery that the sirtuin family members SIRT3, SIRT4, and SIRT5 localize to the mitochondrial matrix has strongly suggested that acetylation could also play an important regulatory role within this organelle $[39,40]$. An early study by Shi et al. supported this suggestion by demonstrating that SIRT3 regulates adaptive responses to cold exposure in brown adipose tissue by increasing the expression of mitochondrial uncoupling protein-1 (UCP1) and thereby increasing mitochondrial respiration [58]. However, the data in this study were generated with a SIRT3 construct lacking a mitochondrial targeting sequence, and, thus, it is not clear whether the results are due to SIRT3 acting within the mitochondria. Soon after this study was published, Schwer et al. and Hallows et al. independently confirmed that mammalian SIRT3 directly regulates the activity of the mitochondrial protein acetyl-CoA synthetase 2 (AceCS2) via $\mathrm{NAD}^{+}$-dependent deacetylation $[59,60]$. These findings demonstrated an 
ancient metabolic regulatory mechanism conserved from the prokaryotic bacterium Salmonella enterica to mammals [61]. Taken together, these early studies not only confirmed reversible $\mathrm{N}^{\varepsilon}$-acetylation as an important regulatory mark within mitochondria but also implicated the sirtuins as key nutrient-responsive modulators of the functional changes acetylation confers upon its target proteins.

The initial studies of mitochondrial acetylation gave way to global proteomics surveys. A 2006 proteomics survey conducted by Kim et al. revealed that over $20 \%$ of liver mitochondrial proteins and enzymes are acetylated and that many change acetylation states in response to acute fasting [2]. Furthermore, enzymes of the major mitochondrial carbon conversion pathways such as the TCA cycle and $\beta$ oxidation were found to be particularly enriched in acetyl modifications. These findings suggested that reversible acetylation could serve as a finely-tuned mechanism for globally regulating the use and conversion of carbon energy sources. The authors also demonstrated that a number of the mitochondrial enzymes they found to be acetylated have known implications in processes of mammalian aging and longevity, such as manganese superoxide dismutase (MnSOD) and NADH:ubiquinone oxidoreductase (respiratory complex I) [2]. Complementing the discovery that global acetylation profiles can change in response to nutrient availability, Schwer et al. showed that global mitochondrial acetylation profiles change in multiple organ systems in response to long-term caloric restriction (CR) [62]. Surprisingly, this study found that the vast majority of mitochondrial enzymes exhibited increases in acetylation during caloric restriction, which counters the belief that sirtuin-mediated deacetylation mediates the beneficial effects of caloric restriction. This finding could suggest that mitochondrial sirtuin-mediated deacetylation plays a relatively minor role in the metabolic changes that accompany caloric restriction and that there are more complex and undiscovered adaptive mechanisms at play. Additionally, Wang et al. and Zhao et al. further confirmed the regulatory importance of acetyl-modifications in metabolism and in the coordination of cellular carbon utilization $[3,63]$. These proteomic surveys have exposed the prevalence of acetylation within mitochondria and reinforced its importance as a regulatory modification. Ongoing studies that aimed at understanding the mechanisms of acetylation dynamics are likely to reveal this as an essential component of mitochondrial biology.

Further focused investigations of acetylation within mitochondria have concentrated on the role of mitochondrial sirtuins. By generating SIRT3, SIRT4, and SIRT5 systemic knockout mouse models, Lombard et al. demonstrated that mice lacking SIRT3 exhibit global hyperacetylation of mitochondrial proteins in liver and brown adipose tissue, whereas the acetylation states in mice lacking SIRT4 and SIRT5 were relatively unchanged [64]. This evidence suggested that SIRT3 primarily functions as a deacetylase and potentially targets a wide range of mitochondrial proteins, whereas the deacetylase activities of SIRT4 and SIRT5 are much more restricted.

Although it is widely accepted that SIRT3's functional role is predominantly within mitochondria, several studies have explored potential nuclear and cytosolic roles for this sirtuin family member. The study mentioned earlier conducted by Shi et al. suggested that SIRT3 can regulate the expression of the nuclear-encoded genes coding for mitochondrial uncoupling protein 1 and peroxisome proliferatoractivated receptor gamma coactivator 1 -alpha (PGC- $1 \alpha$ ) [58]. Similarly, SIRT3 has also been found to regulate the expression of catalase and manganese superoxide dismutase in the heart [65]. Yet another study indicated that SIRT3 can indirectly regulate the association between the glycolytic enzyme hexokinase II and the mitochondria [66]. These studies have formed the basis for future work investigating potential roles for SIRT3 outside of the mitochondria.

Early characterization of SIRT3 null mice indicated that they were phenotypically unremarkable, despite a significant reduction in their liver, heart, and kidney ATP content associated with hyperacetylation and reduced activity of mitochondrial respiratory complex I [7]. A more thorough investigation, however, revealed that SIRT3 ${ }^{-1-}$ mice display hepatic lipid accumulation during fasting resembling human disorders of fatty acid oxidation [4, 67]. This study found that SIRT3 directly deacetylates and activates longchain acyl-CoA dehydrogenase (LCAD), illustrating a critical mechanism for this sirtuin in the metabolic adaptations to fasting and nutrient restriction [4]. Consistent with SIRT3's role in oxidative metabolism, Shulga et al. demonstrated that SIRT3 can stimulate oxidative phosphorylation and a shift away from glycolysis via inactivation of cyclophilin D, which consequently destabilizes the association of hexokinase II with the mitochondria [66]. Furthermore, mitochondrial isocitrate dehydrogenase (IDH2) is deacetylated and activated by SIRT3, thereby regulating a key step in the TCA cycle as well as the supply of NADPH necessary for mitochondrial antioxidant defense mechanisms $[5,68]$. Additional studies have exposed SIRT3 as a regulator of protein translation via deacetylation of the mitochondrial ribosome and in the activity of respiratory complex II (succinate dehydrogenase) $[69,70]$. It is now clear that SIRT3 can influence the activity of oxidative phosphorylation, fatty acid oxidation, and the TCA cycle, positioning this particular sirtuin as a major player in the regulation of intermediary metabolism.

Studies of mitochondrial SIRT4 and SIRT5 are less extensive than those for SIRT3. Earlier characterization of mammalian SIRT4 revealed that it chiefly functions as an $\mathrm{NAD}^{+}$-dependent mono-ADP-ribosyltransferase that specifically ADP-ribosylates and inactivates mitochondrial glutamate dehydrogenase (GDH), thereby regulating amino acid-induced insulin secretion in pancreatic $\beta$ cells $[71,72]$. Later studies also identified GDH as a target of SIRT3mediated activation via deacetylation, but potential crosstalk with SIRT4-mediated ADP ribosylation has not been explored $[5,64]$. Recently, it was demonstrated in vivo that adenoviral-mediated shRNA knockdown of SIRT4 in mouse liver increases the expression of genes involved in fatty acid metabolism [73]. Together, these lines of evidence suggest that SIRT4 could play an important role in the disruption of lipid homeostasis associated with insulin resistance in type 2 diabetes. Further characterization of mitochondrial SIRT5 has shown that it regulates the first 
step in the detoxification of ammonia during the urea cycle via deacetylation-mediated activation of carbamoylphosphate synthetase 1 [6]. Accordingly, SIRT5 ${ }^{-/-}$mice develop hyperammonemia during metabolic states demanding greater protein catabolism such as fasting and caloric restriction $[6,74]$. Another investigation has suggested that SIRT5 can deacetylate cytochrome $c$, a protein involved in mitochondrial respiration and apoptosis [5]. However, the functional importance of this relationship has not been determined and is also inconsistent with findings in the previously mentioned study indicating that SIRT5 is localized to the mitochondrial matrix. Future studies of mitochondrial sirtuins 4 and 5 are likely to elucidate their full regulatory capacity in response to various cellular stresses and stimuli.

Interestingly, other studies have implicated reversible mitochondrial acetylation in mechanisms of cell growth and survival. An early investigation of SIRT3's function in an epithelial cancer cell line indicated that it is required for JNK-regulated cell death via silencing the apoptotic regulator B-cell lymphoma 2 (bcl-2) [75]. In contrast to its role in epithelial cancer, SIRT3 was shown to serve a prosurvival function in cardiomyocytes via deacetylating Ku70 and promoting the sequestration of the proapoptotic protein Bax [43]. In addition to its apoptotic functions, SIRT3 was more recently demonstrated to be a bona fide tumor suppressor by Kim et al. [76]. This study showed that SIRT3 ${ }^{-1-}$ mouse embryonic fibroblasts display reduced antioxidant defenses, which result in a lower threshold for tumorigenesis - a finding that confirmed earlier work that established SIRT3's involvement in the transcriptional regulation of antioxidant genes $[65,77]$. Furthermore, the transformation-permissive effect of SIRT3 ablation can be reversed upon the addition of exogenous MnSOD [76]. Consistent with these findings, two more recently published investigations independently confirmed that SIRT3 directly deacetylates and activates MnSOD, thereby linking nutrient availability to antioxidant defenses $[78,79]$. A different study conducted by Yang et al. established that the levels of mitochondrial $\mathrm{NAD}^{+}$are a critical determinant to cell survival [80]. Mitochondrial $\mathrm{NAD}^{+}$levels were shown to be regulated by the rate-limiting enzyme in the mammalian $\mathrm{NAD}^{+}$-salvage pathway, nicotinamide phosphoribosyltransferase (Nampt). Importantly, the authors demonstrated that $\mathrm{NAD}^{+}$-responsive mitochondrial SIRT3 and SIRT4 are required for improved cell survival when mitochondrial $\mathrm{NAD}^{+}$levels rise under conditions of fasting and induced genotoxic stress [80]. Recently, two independent approaches also implicated SIRT3 in the regulation of p53-mediated growth arrest $[81,82]$. Taken together, these studies indicate that mitochondrial SIRT3 is a critical mediator of cell growth and survival which may hold therapeutic implications in the treatment of various cancers.

There are also several interesting acetylated mitochondrial proteins involved in cellular stress responses and apoptosis on which the effect of reversible acetylation has not been studied. These proteins include apoptosisinducing factor (AIF) responsible for DNA fragmentation during apoptosis, cytochrome $c$ which forms an important component of the apoptosome as well as voltage-dependent anion channel (VDAC) and adenine nucleotide translocase (ANT), both of which are believed to form the mitochondrial permeability transition pore during apoptosis $[2,83]$. Furthermore, all of the previously mentioned mitochondrial proteins are reversibly acetylated in a nutrient-sensitive manner, suggesting that the apoptotic functions of these proteins may be regulated by cellular energy status [2]. The study of mitochondrial acetylation states in processes of cell death and survival represents an entirely open field of study that is rife with intriguing biological questions that could provide a new layer of understanding to the mechanisms and regulation of apoptosis and cell growth.

The majority of the studies in the field of mitochondrial acetylation thus far have focused on the mitochondrial localized members of the sirtuin family of deacetylases, while the search for the counterbalancing mitochondrial acetyltransferase(s) has so far been unsuccessful. Although it is possible that certain nuclear-encoded, mitochondrial targeted proteins acquire acetylation marks prior to mitochondrial import, the confirmed acetylation of the mitochondrial confined ATP synthase subunit 8 protein makes the existence of a mitochondrial acetyltransferase(s) highly likely [2, 83]. The marked enrichment of acetyl-modifications occurring on enzymes involved in major processes such as $\beta$-oxidation and redox chemistry ( $64 \%$ and $44 \%$, resp.) over the total pool of acetylated mitochondrial proteins (22\%) also suggests finely tuned enzymatic mechanisms for regulating carbon flux within mitochondria [2]. Furthermore, the amino acid sequence motifs favoring acetylation within mitochondria differ from those of the nucleus and cytosol, suggesting a mitochondrial-specific acetyltransferase(s) possessing its own distinct set of substrates [2]. Despite the strong evidence for a mitochondrial acetyltransferase(s), it is also important to consider the possibility of nonenzymatic acetylation occurring in response to changes in the overall mitochondrial acetyl-CoA pool. Indeed, nonenzymatic acetylation is known to occur on histone substrates and may account for the unexpected increases in mitochondrial acetylation in response to caloric restriction $[62,84]$.

How do large changes in the acetylation state of a given mitochondrial protein pool affect protein function? Furthermore, how do large-scale changes in global mitochondrial acetylation states influence mitochondrial biology and overall cellular energy status? Although little work has been conducted to address these questions, some insights can be drawn from the literature. In the work of Hirschey et al., the authors performed semiquantitative mass spectrometry of the fatty acid oxidation protein LCAD in wild-type and SIRT3 ${ }^{-/-}$livers in an effort to identify differentially acetylated lysine residues of interest [4]. The analysis discovered eight acetylation sites on LCAD; however, only one particular acetyl-lysine residue, K42, was found to be critically important for the SIRT3-dependent modulation of LCAD's enzymatic activity. Constitutively mimicking deacetylation at K42 induced a nearly $60 \%$ increase in LCAD activity. Furthermore, acetylation at only K42 accounted for over $70 \%$ of the total LCAD acetyl-lysine signal in SIRT3 $^{-/-}$liver mitochondria as quantified by Western blot 
densitometry. Earlier work performed by this group found a similar pattern with K642 of the mitochondrial enzyme acetyl-CoA synthetase 2 [59]. These findings suggest that large changes in acetylation signal and protein function could be induced by relatively limited or restricted changes in the acetylation state of a given protein. Accordingly, global changes in mitochondrial acetylation, such as those observed in the SIRT3 ${ }^{-1-}$ mitochondria, might represent an unexpectedly limited number of acetylation state changes corresponding to large functional and metabolic consequences. Future work in this field will surely provide exciting insights into these questions, and the imminent discovery of the mitochondrial acetyltransferases(s) or alternative acetylation mechanisms will provide a critical step toward a more complete understanding of mitochondrial acetylation biology and its implications for disease states.

\section{Mitochondrial Acetylation and Heart Failure}

The recently understood prevalence of acetyl modifications within the mitochondria that can potentially modulate processes of intermediary metabolism suggests that altered acetylation could potentially contribute to age-associated disease states in which there are recognized alterations in mitochondrial carbon utilization, such as heart failure and cancer.

The unfortunate progression from increased cardiac workload to pathological cardiac hypertrophy and, ultimately, heart failure occurs when the long-term metabolic demands of the body exceed the ability of the pumping heart to meet them. Although there are a number of human conditions capable of leading to heart failure including renal insufficiency, high blood pressure, and cardiac arrhythmias, the leading risk factor is increasing age [85]. The role of mitochondrial dysfunction in heart failure and the aging process in general is well established. Mitochondrial disorders are often characterized by myopathies of the heart and skeletal muscle, as these highly energetic organs are heavily dependent on mitochondria-derived energy generation [86]. Furthermore, cardiomyopathy and heart failure in the general population are often regarded as diseases of impaired energy homeostasis associated with a depletion of the cardiomyocyte ATP and creatine pools [87, 88]. Interestingly, mice lacking the predominant mitochondrial $\mathrm{NAD}^{+}$-dependent deacetylase SIRT3 also exhibit depleted cardiac ATP levels and further display global hyperacetylation of mitochondrial proteins in liver and brown adipose tissue $[7,64]$. These data suggest a role for mitochondrial SIRT3 dysfunction in the pathophysiology of heart failure. Several additional studies also stand in support of this notion. As discussed earlier, Sundaresan et al. have shown that SIRT3 promotes cardiomyocyte cell survival during genotoxic and oxidative stress [43]. This group has also revealed that SIRT3 transgenic mice are resistant to agonist-induced cardiac hypertrophy via SIRT3-mediated upregulation of antioxidant genes [65]. Moreover, exogenous administration of $\mathrm{NAD}^{+}$into the hearts of mice was found to abrogate agonist-induced cardiac hypertrophy in a SIRT3-dependent manner [89]. Similarly, transgenic mice harboring cardiac-specific overexpression of the $\mathrm{NAD}^{+}$salvaging enzyme Nampt display reduced infarct size following ischemia-reperfusion injury, raising the possibility that the cardioprotective effect of increased $\mathrm{NAD}^{+}$is mediated by the mitochondrial sirtuins [90]. Pathophysiological cardiac remodeling and heart failure in humans are also known to develop in response to alterations in the renin-angiotensin system (RAS) [91]. One of the RAS signaling proteins, angiotensin II, is often used to induce hypertension and cardiac hypertrophy in experimental animals. Interestingly, ablation of the angiotensin II type I receptor in mice reduces cardiac injury, increases lifespan, and upregulates the expression of SIRT3 and its upstream regulator Nampt in the kidney [92]. Collectively, these studies implicate the mitochondrial deacetylase SIRT3 as a major cardioprotective enzyme, the modulation of which may hold therapeutic promise in the treatment of cardiac disease.

Mitochondrial oxidative phosphorylation is responsible for generating over $90 \%$ of the energy-rich ATP in the adult human heart $[93,94]$. Under basal conditions, as much as $70 \%$ of this ATP is derived from fatty acid $\beta$ oxidation, and the remainder is generated from the oxidation of glucose, lactate, and ketone bodies [95]. The energetic substrate preferences of the heart can change in response to acute stimuli or stressors such as exercise, energy demand, hormonal regulation, ischemia, and substrate availability $[95,96]$. Thus, the healthy heart is a versatile and adaptive consumer of energy substrates. The failing heart, however, experiences complex alterations in energy metabolism and substrate utilization that are incompletely understood [97]. Studies on rodent and canine models generally indicate that the failing myocardium suffers downregulation of mitochondrial respiratory chain complexes, decreased oxygen consumption and ATP generation, decreased flux through the creatine kinase system, and impaired fatty acid oxidation [95, 97-100]. Many have proposed targeting these metabolic rearrangements and altering substrate utilization as a therapeutic strategy to treat heart failure [101]. Interestingly, numerous mitochondrial enzymes of the respiratory chain and those involved in the oxidation of glucose and fat carry one or more acetylation marks that are reversible in a nutrient-sensitive manner. These include respiratory complexes I, II, and V, pyruvate dehydrogenase, several acylCoA dehydrogenases, and carnitine palmitoyltransferase 1 and 2, among many others $[2,62,83]$. It has also been demonstrated that the mitochondrial deacetylase SIRT3 can directly promote oxidative metabolism via deacetylationmediated activation of respiratory complex 1, long-chain acyl-CoA dehydrogenase (LCAD), and cyclophilin D as discussed earlier $[4,7,66]$. The notion that acetylation within the mitochondria can modulate the activity of metabolic enzymes implies that there may be altered acetylation states within the mitochondria and that they could contribute to the pathophysiology of heart failure. More importantly, if this is true, devising strategies that directly target mitochondrial acetylation may provide therapeutic benefit in pathological rearrangements of cardiac energy metabolism. 


\section{Mitochondrial Acetylation and Cancer}

In 1924, Warburg and Negelein made the seminal observation that many cancer cells prefer to metabolize glucose into lactate even in the presence of sufficient oxygen to support mitochondrial oxidative phosphorylation [102]. This phenomenon, known as the "Warburg Effect" or aerobic glycolysis, was hypothesized by Warburg to be a defect in the mitochondrial metabolism of tumor cells [103]. In the roughly 70 years following its discovery, little progress was made in uncovering the biochemical mechanisms underlying the propensity of tumor cells to prefer aerobic glycolysis. The last 15 years of oncology research, however, have experienced a renewed interest in understanding the Warburg Effect, and a number of important discoveries have been made. Notably, it has been observed that tumor cell lines almost exclusively express the embryonic isoform of the glycolytic enzyme pyruvate kinase (PKM2), which contributes to the glycolytic preference and lactate production observed in cancer cells [104, 105]. Recent work conducted by Vander Heiden et al. has even provided early evidence of an alternative carbon utilization pathway in tumors expressing PKM2 [106]. Further work on tumor metabolism has revealed that malignant cells also consume large amounts of glutamine in an effort to replenish the TCA cycle intermediates necessary for a high rate of macromolecular synthesis that is characteristic of rapidly proliferating cells [107]. This "glutamine addition" can partially be explained by induction of a transcriptional program that favors glutaminolysis via the activation of the proto-oncogene Myc, which can occur in many forms of human cancers including neuroblastoma, lymphoma, and small cell lung cancer [108-112]. Together, these studies and the recent revitalized interest in cancer bioenergetics have unveiled some key mechanisms underlying the remarkable ability of transformed cells to proliferate. Despite these recent advances, there is still much that we do not understand about a tumor cell's aptitude for reprogramming metabolism to meet its rapid growth demands, and this represents an active area of investigation.

Orchestrating malignant changes in cellular behavior often occurs at the level of posttranslation regulation. Indeed, many cancers are characterized by mutations in, or altered activity of, certain kinases including tumor suppressor LKB1, Src, phosphatidylinositol-3-kinase (PI3K), and the mutant BCR-ABL fusion kinase, which often occurs in chronic myelogenous leukemia (CML) [113-116]. Additionally, there is an appreciated role for cancer-associated transcriptional changes mediated by nuclear KATs and KDACs [26, 117]. Interestingly, the predominant mitochondrial deacetylase SIRT3 has recently been reported to function as a tumor suppressor [76]. This work demonstrated that SIRT3 ${ }^{-1-}$ fibroblasts exogenously transformed with the oncogenes Myc and Ras display reduced activity of mitochondrial respiratory complexes I and III [76]. These results are consistent with earlier work demonstrating direct activation of mitochondrial complex I via SIRT3-mediated deacetylation [7]. Moreover, these findings suggest that there is impaired oxidative phosphorylation and a heavier reliance on glycolytic metabolism in order to satisfy the energetic requirements of transformed SIRT3 ${ }^{-1-}$ fibroblasts, representing the primary hallmarks of the Warburg Effect. Coupling these findings with the knowledge that SIRT3 could potentially be regulating numerous mitochondrial proteins suggests that SIRT3 dysfunction may represent an important factor contributing to the poorly understood metabolic reprogramming that occurs during tumorigenesis [64]. Consistent with this notion, SIRT3 is known to be downregulated in certain breast cancers [76]. Furthermore, if SIRT3 can function as a tumor suppressor, it is logical to conceive that the putative mitochondrial acetyltransferase(s) counterbalancing the tumor-suppressive effects of SIRT3 would be characterized as oncogene(s). A multitude of mitochondrial proteins are now known to be acetylated, many of which are modified in a reversible and nutrientresponsive manner. Accordingly, alterations in mitochondrial acetylation states, and, hence, alterations in carbon substrate utilization, may contribute to the unusual preference for aerobic glycolysis and glutaminolysis often observed in numerous forms of cancer.

In addition to understanding the emerging importance of mitochondrial acetylation in age-associated diseases, it is also important to briefly discuss evidence for its involvement in the aging process in general. Heart failure and cancer are two pathophysiological states that display an exponentially increasing incidence with advancing age, and a role for the mitochondrial deacetylase SIRT3 in these respective conditions has been discussed [85]. Complementing SIRT3's emerging role in diseases of aging is the interesting though controversial observation that SIRT3 is the only member of the sirtuin family reportedly linked to longevity in humans. An early study of the human SIRT3 gene identified a guanine to thymine (G477T) single nucleotide polymorphism (SNP) located in exon three to be associated with survivorship in elderly males [118]. Later, Bellizzi et al. discovered a variable number of tandem repeat (VNTR) polymorphisms possessing enhancer activity at the SIRT3 locus [119]. The authors of this study further demonstrated that the inactive form of the SIRT3 enhancer is underrepresented in a population exceeding 90 years of age, and thus concluded that the active VNTR enhancer is associated with longevity. Additional work has identified the activating elements that bind to this intronic VNTR enhancer sequence [120]. In contrast to these studies, a meta-analysis of SIRT3 SNP data coupled with a larger association study of the SIRT3 chromosomal region in centenarians indicated no significant genomic variation that could be linked to longevity, with the exception of one possible SNP, rs939915 [121]. Future analysis of genomic variation at the mammalian SIRT3 locus, transgenic animals, and functional studies of SIRT3 and its deacetylation targets will provide much-needed insight into the possible role that this sirtuin plays in longevity and the aging process.

\section{Summary}

Although the acetylation of lysine residues has been known as a posttranslational modification for over 40 years, it was not until the last 15 years that advances in technology 
and molecular techniques have enabled the rigorous study of this highly interesting regulatory mechanism. We now understand that lysine acetylation rivals phosphorylation, in respect to both the sheer number of acetylated proteins in mammalian cells as well as in its regulatory capacity. Furthermore, global cellular acetylation states are largely dependent on mitochondrial-derived processes of acetylCoA formation. Many proteins within the mitochondria, itself, are now known to be reversibly acetylated and, in some cases, acetylation can regulate metabolism and the fate of carbon energy sources. Thus, reversible acetylation of mitochondrial proteins represents a new layer of protein regulation mediating acute and adaptive changes in mammalian metabolism. This new framework for understanding metabolism suggests that altered mitochondrial acetylation patterns can contribute to the pathological shifts in energy generation that are known to occur during heart failure, the rapid proliferation of malignant tumor cells, and, indeed, the aging process in general. It is also worth noting that mitochondrial acetylation in neurodegenerative pathologies is currently an unstudied area. Mitochondrial acetylation biology is an infant field of study that, in the future, will have the potential to bridge our understanding of cellular metabolism, cell death and survival, and the inevitable pathological consequences of aging.

\section{Acknowledgments}

The authors would like to acknowledge Kyle Martin and Cliff Babbey for their thoughtful review of the figures and manuscript and Christopher Brown of the Indiana University School of Medicine's Visual Media Department for the kind preparation of Figure 1. Support for this project was provided in part by the National Institutes of Health 1P01HL 085098 and the Friedreich's Ataxia Research Alliance.

\section{References}

[1] B. D. Strahl and C. D. Allis, "The language of covalent histone modifications," Nature, vol. 403, no. 6765, pp. 41-45, 2000.

[2] S. C. Kim, R. Sprung, Y. Chen et al., "Substrate and functional diversity of lysine acetylation revealed by a proteomics survey," Molecular Cell, vol. 23, no. 4, pp. 607-618, 2006.

[3] Q. Wang, Y. Zhang, C. Yang et al., "Acetylation of metabolic enzymes coordinates carbon source utilization and metabolic flux," Science, vol. 327, no. 5968, pp. 1004-1007, 2010.

[4] M. D. Hirschey, T. Shimazu, E. Goetzman et al., "SIRT3 regulates mitochondrial fatty-acid oxidation by reversible enzyme deacetylation," Nature, vol. 464, no. 1, pp. 121-125, 2010.

[5] C. Schlicker, M. Gertz, P. Papatheodorou, B. Kachholz, C. F. W. Becker, and C. Steegborn, "Substrates and regulation mechanisms for the human mitochondrial sirtuins Sirt3 and Sirt5," Journal of Molecular Biology, vol. 382, no. 3, pp. 790801, 2008.

[6] T. Nakagawa, D. J. Lomb, M. C. Haigis, and L. Guarente, "SIRT5 deacetylates carbamoyl phosphate synthetase 1 and regulates the urea cycle," Cell, vol. 137, no. 3, pp. 560-570, 2009.
[7] B. H. Ahn, H. S. Kim, S. Song et al., "A role for the mitochondrial deacetylase Sirt3 in regulating energy homeostasis," Proceedings of the National Academy of Sciences of the United States of America, vol. 105, no. 38, pp. 14447-14452, 2008.

[8] L. Guarente, "Calorie restriction and SIR2 genes-towards a mechanism," Mechanisms of Ageing and Development, vol. 126, no. 9, pp. 923-928, 2005.

[9] M. C. Haigis and L. P. Guarente, "Mammalian sirtuinsemerging roles in physiology, aging, and calorie restriction," Genes and Development, vol. 20, no. 21, pp. 2913-2921, 2006.

[10] H. A. Krebs, "The citric acid cycle and the Szent-Györgyi cycle in pigeon breast muscle," Biochemical Journal, vol. 34, no. 5, pp. 775-779, 1940.

[11] P. Mitchell, "Coupling of phosphorylation to electron and hydrogen transfer by a chemi-osmotic type of mechanism," Nature, vol. 191, no. 4784, pp. 144-148, 1961.

[12] D. E. Bauer, G. Hatzivassiliou, F. Zhao, C. Andreadis, and C. B. Thompson, "ATP citrate lyase is an important component of cell growth and transformation," Oncogene, vol. 24, no. 41, pp. 6314-6322, 2005.

[13] K. Bloch, "The biological synthesis of cholesterol," Science, vol. 150, no. 3692, pp. 19-28, 1965.

[14] P. A. Edwards and J. Ericsson, "Sterols and isoprenoids: signaling molecules derived from the cholesterol biosynthetic pathway," Annual Review of Biochemistry, vol. 68, pp. 157185, 1999.

[15] R. Nosadini, A. Avogaro, A. Doria, P. Fioretto, R. Trevisan, and A. Morocutti, "Ketone body metabolism: a physiological and clinical overview," Diabetes/Metabolism Reviews, vol. 5, no. 3, pp. 299-319, 1989.

[16] A. Luong, V. C. Hannah, M. S. Brown, and J. L. Goldstein, "Molecular characterization of human acetyl-CoA synthetase, an enzyme regulated by sterol regulatory elementbinding proteins," Journal of Biological Chemistry, vol. 275, no. 34, pp. 26458-26466, 2000.

[17] T. Fujino, J. Kondo, M. Ishikawa, K. Morikawa, and T. T. Yamamoto, "Acetyl-CoA synthetase 2, a mitochondrial matrix enzyme involved in the oxidation of acetate," Journal of Biological Chemistry, vol. 276, no. 14, pp. 11420-11426, 2001.

[18] I. Sakakibara, T. Fujino, M. Ishii et al., "Fasting-induced hypothermia and reduced energy production in mice lacking acetyl-CoA synthetase 2," Cell Metabolism, vol. 9, no. 2, pp. 191-202, 2009.

[19] K. E. Wellen, G. Hatzivassiliou, U. M. Sachdeva, T. V. Bui, J. R. Cross, and C. B. Thompson, "ATP-citrate lyase links cellular metabolism to histone acetylation," Science, vol. 324, no. 5930, pp. 1076-1080, 2009.

[20] E. L. Gershey, G. Vidali, and V. G. Allfrey, "Chemical studies of histone acetylation. The occurrence of epsilon$\mathrm{N}$-acetyllysine in the f2al histone," Journal of Biological Chemistry, vol. 243, no. 19, pp. 5018-5022, 1968.

[21] A. Inoue and D. Fujimoto, "Enzymatic deacetylation of histone," Biochemical and Biophysical Research Communications, vol. 36, no. 1, pp. 146-150, 1969.

[22] D. Fujimoto and K. Segawa, "Enzymatic deacetylation of f2a2 histone,” FEBS Letters, vol. 32, no. 1, pp. 59-61, 1973.

[23] A. Kervabon, J. Mery, and J. Parello, "Enzymatic deacetylation of a synthetic peptide fragment of histone H4," FEBS Letters, vol. 106, no. 1, pp. 93-96, 1979.

[24] J. Taunton, C. A. Hassig, and S. L. Schreiber, "A mammalian histone deacetylase related to the yeast transcriptional regulator Rpd3p," Science, vol. 272, no. 5260, pp. 408-411, 1996. 
[25] T. Jenuwein and C. D. Allis, "Translating the histone code," Science, vol. 293, no. 5532, pp. 1074-1080, 2001.

[26] A. Copeland, D. Buglio, and A. Younes, "Histone deacetylase inhibitors in lymphoma," Current Opinion in Oncology, vol. 22, no. 5, pp. 431-436, 2010.

[27] I. V. Gregoretti, Y. M. Lee, and H. V. Goodson, "Molecular evolution of the histone deacetylase family: functional implications of phylogenetic analysis," Journal of Molecular Biology, vol. 338, no. 1, pp. 17-31, 2004.

[28] C. D. Allis, S. L. Berger, J. Cote et al., "New nomenclature for chromatin-modifying enzymes," Cell, vol. 131, no. 4, pp. 633-636, 2007.

[29] A. J. M. de Ruijter, A. H. van Gennip, H. N. Caron, S. Kemp, and A. B. P. van Kuilenburg, "Histone deacetylases (HDACs): characterization of the classical HDAC family," Biochemical Journal, vol. 370, no. 3, pp. 737-749, 2003.

[30] A. J. Bannister and E. A. Miska, "Regulation of gene expression by transcription factor acetylation," Cellular and Molecular Life Sciences, vol. 57, no. 8-9, pp. 1184-1192, 2000.

[31] W. Gu and R. G. Roeder, "Activation of p53 sequence-specific DNA binding by acetylation of the p53 C-terminal domain," Cell, vol. 90, no. 4, pp. 595-606, 1997.

[32] M. A. Glozak, N. Sengupta, X. Zhang, and E. Seto, "Acetylation and deacetylation of non-histone proteins," Gene, vol. 363, no. 1-2, pp. 15-23, 2005.

[33] P. Bali, M. Pranpat, J. Bradner et al., "Inhibition of histone deacetylase 6 acetylates and disrupts the chaperone function of heat shock protein 90: a novel basis for antileukemia activity of histone deacetylase inhibitors," Journal of Biological Chemistry, vol. 280, no. 29, pp. 26729-26734, 2005.

[34] T. Sekimoto, T. Matsuyama, T. Fukui, and K. Tanizawa, "Evidence for lysine 80 as general base catalyst of leucine dehydrogenase," Journal of Biological Chemistry, vol. 268, no. 36, pp. 27039-27045, 1993.

[35] W. Yu, Y. Lin, J. Yao et al., "Lysine 88 acetylation negatively regulates ornithine carbamoyltransferase activity in response to nutrient signals," Journal of Biological Chemistry, vol. 284, no. 20, pp. 13669-13675, 2009.

[36] Z. L. Yuan, Y. J. Guan, D. Chatterjee, and Y. E. Chin, "Stat3 dimerization regulated by reversible acetylation of a single lysine residue," Science, vol. 307, no. 5707, pp. 269-273, 2005.

[37] K. Sadoul, C. Boyault, M. Pabion, and S. Khochbin, "Regulation of protein turnover by acetyltransferases and deacetylases," Biochimie, vol. 90, no. 2, pp. 306-312, 2008.

[38] X. J. Yang and E. Seto, "Lysine acetylation: codified crosstalk with other posttranslational modifications," Molecular Cell, vol. 31, no. 4, pp. 449-461, 2008.

[39] P. Onyango, I. Celic, J. M. McCaffery, J. D. Boeke, and A. P. Feinberg, "SIRT3, a human SIR2 homologue, is an NAD-dependent deacetylase localized to mitochondria," Proceedings of the National Academy of Sciences of the United States of America, vol. 99, no. 21, pp. 13653-13658, 2002.

[40] E. Michishita, J. Y. Park, J. M. Burneskis, J. C. Barrett, and I. Horikawa, "Evolutionarily conserved and nonconserved cellular localizations and functions of human SIRT proteins," Molecular Biology of the Cell, vol. 16, no. 10, pp. 4623-4635, 2005.

[41] K. Aquilano, P. Vigilanza, S. Baldelli, B. Pagliei, G. Rotilio, and M. R. Ciriolo, "Peroxisome proliferator-activated receptor $\gamma$ co-activator $1 \alpha(\mathrm{PGC}-1 \alpha)$ and sirtuin 1 (SIRT1) reside in mitochondria: possible direct function in mitochondrial biogenesis," Journal of Biological Chemistry, vol. 285, no. 28, pp. 21590-21599, 2010.
[42] R. Amat, A. Planavila, S. L. Chen, R. Iglesias, M. Giralt, and F. Villarroya, "SIRT1 controls the transcription of the peroxisome proliferator-activated receptor- $\gamma$ co-activator$1 \alpha(\mathrm{PGC}-1 \alpha)$ gene in skeletal muscle through the PGC- $1 \alpha$ autoregulatory loop and interaction with MyoD," Journal of Biological Chemistry, vol. 284, no. 33, pp. 21872-21880, 2009.

[43] N. R. Sundaresan, S. A. Samant, V. B. Pillai, S. B. Rajamohan, and M. P. Gupta, "SIRT3 is a stress-responsive deacetylase in cardiomyocytes that protects cells from stress-mediated cell death by deacetylation of Ku70," Molecular and Cellular Biology, vol. 28, no. 20, pp. 6384-6401, 2008.

[44] A. Kaidi, B. T. Weinert, C. Choudhary, and S. P. Jackson, "Human SIRT6 promotes DNA end resection through CtIP deacetylation," Science, vol. 329, no. 5997, pp. 1348-1353, 2010.

[45] E. M. Dioum, R. Chen, M. S. Alexander et al., "Regulation of hypoxia-inducible factor $2 \alpha$ signaling by the stressresponsive deacetylase sirtuin 1," Science, vol. 324, no. 5932, pp. 1289-1293, 2009.

[46] H.-S. Kim, C. Xiao, R.-H. Wang et al., "Hepatic-specific disruption of SIRT6 in mice results in fatty liver formation due to enhanced glycolysis and triglyceride synthesis," Cell Metabolism, vol. 12, no. 3, pp. 224-236, 2010.

[47] A. A. Sauve, C. Wolberger, V. L. Schramm, and J. D. Boeke, "The biochemistry of sirtuins," Annual Review of Biochemistry, vol. 75, pp. 435-465, 2006.

[48] T. Yang and A. A. Sauve, "NAD metabolism and sirtuins: metabolic regulation of protein deacetylation in stress and toxicity," AAPS Journal, vol. 8, no. 4, pp. E632-E643, 2006.

[49] J. C. Jiang, E. Jaruga, M. V. Repnevskaya, and S. M. Jazwinski, "An intervention resembling caloric restriction prolongs life span and retards aging in yeast," FASEB Journal, vol. 14, no. 14, pp. 2135-2137, 2000.

[50] W. Mair, P. Goymer, S. D. Pletcher, and L. Partridge, "Demography of dietary restriction and death in Drosophila," Science, vol. 301, no. 5640, pp. 1731-1733, 2003.

[51] J. Mattison, G. Roth, M. Lane, and D. Ingram, "Dietary restriction in aging nonhuman primates," Interdisciplinary Topics in Gerontology, vol. 35, pp. 137-158, 2007.

[52] R. Weindruch and R. L. Walford, "Dietary restriction in mice beginning at 1 year of age: effect on life-span and spontaneous cancer incidence," Science, vol. 215, no. 4538, pp. 1415-1418, 1982.

[53] J. C. Milne and J. M. Denu, "The Sirtuin family: therapeutic targets to treat diseases of aging," Current Opinion in Chemical Biology, vol. 12, no. 1, pp. 11-17, 2008.

[54] H. Daub, J. V. Olsen, M. Bairlein et al., "Kinase-selective enrichment enables quantitative phosphoproteomics of the kinome across the cell cycle," Molecular Cell, vol. 31, no. 3, pp. 438-448, 2008.

[55] D. Cantu, J. Schaack, and M. Patel, "Oxidative inactivation of mitochondrial aconitase results in iron and $\mathrm{H}_{2} \mathrm{O}_{2}$ neurotoxicity in rat primary mesencephalic cultures," PLoS One, vol. 4, no. 9, Article ID e7095, 2009.

[56] T.-I. Peng and M.-J. Jou, "Oxidative stress caused by mitochondrial calcium overload," Annals of the New York Academy of Sciences, vol. 1201, pp. 183-188, 2010.

[57] D. Blache, S. Devaux, O. Joubert et al., "Long-term moderate magnesium-deficient diet shows relationships between blood pressure, inflammation and oxidant stress defense in aging rats," Free Radical Biology and Medicine, vol. 41, no. 2, pp. 277-284, 2006.

[58] T. Shi, F. Wang, E. Stieren, and Q. Tong, "SIRT3, a mitochondrial sirtuin deacetylase, regulates mitochondrial 
function and thermogenesis in brown adipocytes," Journal of Biological Chemistry, vol. 280, no. 14, pp. 13560-13567, 2005.

[59] B. Schwer, J. Bunkenborg, R. O. Verdin, J. S. Andersen, and E. Verdin, "Reversible lysine acetylation controls the activity of the mitochondrial enzyme acetyl-CoA synthetase 2," Proceedings of the National Academy of Sciences of the United States of America, vol. 103, no. 27, pp. 10224-10229, 2006.

[60] W. C. Hallows, S. Lee, and J. M. Denu, "Sirtuins deacetylate and activate mammalian acetyl-CoA synthetases," Proceedings of the National Academy of Sciences of the United States of America, vol. 103, no. 27, pp. 10230-10235, 2006.

[61] V. J. Starai, I. Celic, R. N. Cole, J. D. Boeke, and J. C. Escalante-Semerena, "Sir2-dependent activation of acetylCoA synthetase by deacetylation of active lysine," Science, vol. 298, no. 5602, pp. 2390-2392, 2002.

[62] B. Schwer, M. Eckersdorff, Y. Li et al., "Calorie restriction alters mitochondrial protein acetylation," Aging Cell, vol. 8, no. 5, pp. 604-606, 2009.

[63] S. Zhao, W. Xu, W. Jiang et al., "Regulation of cellular metabolism by protein lysine acetylation," Science, vol. 327, no. 5968, pp. 1000-1004, 2010.

[64] D. B. Lombard, F. W. Alt, H. L. Cheng et al., "Mammalian Sir2 homolog SIRT3 regulates global mitochondrial lysine acetylation," Molecular and Cellular Biology, vol. 27, no. 24, pp. 8807-8814, 2007.

[65] N. R. Sundaresan, M. Gupta, G. Kim, S. B. Rajamohan, A. Isbatan, and M. P. Gupta, "Sirt3 blocks the cardiac hypertrophic response by augmenting Foxo3a-dependent antioxidant defense mechanisms in mice," Journal of Clinical Investigation, vol. 119, no. 9, pp. 2758-2771, 2009.

[66] N. Shulga, R. Wilson-Smith, and J. G. Pastorino, "Sirtuin3 deacetylation of cyclophilin $\mathrm{D}$ induces dissociation of hexokinase II from the mitochondria," Journal of Cell Science, vol. 123, no. 6, pp. 894-902, 2010.

[67] P. Rinaldo and D. Matern, "Disorders of fatty acid transport and mitochondrial oxidation: challenges and dilemmas of metabolic evaluation," Genetics in Medicine, vol. 2, no. 6, pp. 338-344, 2000.

[68] S. Someya, W. Yu, W. C. Hallows et al., "Sirt3 mediates reduction of oxidative damage and prevention of age-related hearing loss under caloric restriction," Cell, vol. 143, no. 5, pp. 802-812.

[69] Y. Yang, H. Cimen, M. J. Han et al., "NAD" -dependent deacetylase SIRT3 regulates mitochondrial protein synthesis by deacetylation of the ribosomal protein MRPL10," Journal of Biological Chemistry, vol. 285, no. 10, pp. 7417-7429, 2010.

[70] H. Cimen, M. J. Han, Y. Yang, Q. Tong, H. Koc, and E. C. Koc, "Regulation of succinate dehydrogenase activity by SIRT3 in mammalian mitochondria," Biochemistry, vol. 49, no. 2, pp. 304-311, 2010.

[71] M. C. Haigis, R. Mostoslavsky, K. M. Haigis et al., "SIRT4 inhibits glutamate dehydrogenase and opposes the effects of calorie restriction in pancreatic $\beta$ cells," Cell, vol. 126, no. 5, pp. 941-954, 2006.

[72] N. Ahuja, B. Schwer, S. Carobbio et al., "Regulation of insulin secretion by SIRT4, a mitochondrial ADPribosyltransferase," Journal of Biological Chemistry, vol. 282, no. 46, pp. 33583-33592, 2007.

[73] N. Nasrin, X. Wu, E. Fortier et al., "SIRT4 regulates fatty acid oxidation and mitochondrial gene expression in liver and muscle cells," Journal of Biological Chemistry, vol. 285, no. 42, pp. 31995-32002, 2010.
[74] T. Nakagawa and L. Guarente, "Urea cycle regulation by mitochondrial sirtuin, SIRT5," Aging, vol. 1, no. 6, pp. 578581, 2009.

[75] S. J. Allison and J. Milner, "SIRT3 is pro-apoptotic and participates in distinct basal apoptotic pathways," Cell Cycle, vol. 6, no. 21, pp. 2669-2677, 2007.

[76] H. S. Kim, K. Patel, K. Muldoon-Jacobs et al., "SIRT3 is a mitochondria-localized tumor suppressor required for maintenance of mitochondrial integrity and metabolism during stress," Cancer Cell, vol. 17, no. 1, pp. 41-52, 2010.

[77] K. M. Jacobs, J. D. Pennington, K. S. Bisht et al., "SIRT3 interacts with the daf-16 homolog FOXO3a in the mitochondria, as well as increases FOXO3a dependent gene expression," International Journal of Biological Sciences, vol. 4, no. 5, pp. 291-299, 2008.

[78] X. Qiu, K. Brown, M. D. Hirschey, E. Verdin, and D. Chen, "Calorie restriction reduces oxidative stress by SIRT3mediated SOD2 activation," Cell Metabolism, vol. 12, no. 6, pp. 662-667, 2010.

[79] R. Tao, M. C. Coleman, J. D. Pennington et al., "Sirt3mediated deacetylation of evolutionarily conserved lysine 122 regulates MnSOD activity in response to stress," Molecular Cell, vol. 40, no. 6, pp. 893-904, 2010.

[80] H. Yang, T. Yang, J. A. Baur et al., "Nutrient-sensitive mitochondrial $\mathrm{NAD}^{+}$levels dictate cell survival," Cell, vol. 130, no. 6, pp. 1095-1107, 2007.

[81] S. Li, M. Banck, S. Mujtaba, M.-M. Zhou, M. M. Sugrue, and M. J. Walsh, "p53-Induced growth arrest is regulated by the Mitochondrial SirT3 deacetylase," PLoS One, vol. 5, no. 5, Article ID e10486, 2010.

[82] Y. Kawamura, Y. Uchijima, N. Horike et al., "Sirt3 protects in vitro-fertilized mouse preimplantation embryos against oxidative stress-induced p53-mediated developmental arrest," Journal of Clinical Investigation, vol. 120, no. 8, pp. 2817-2828, 2010.

[83] C. Choudhary, C. Kumar, F. Gnad et al., "Lysine acetylation targets protein complexes and co-regulates major cellular functions," Science, vol. 325, no. 5942, pp. 834-840, 2009.

[84] W. K. Paik, D. Pearson, H. W. Lee, and S. Kim, "Nonenzymatic acetylation of histones with acetyl-CoA," Biochimica et Biophysica Acta, vol. 213, no. 2, pp. 513-522, 1970.

[85] D. L. Hoyert, M. P. Heron, S. L. Murphy, and H. C. Kung, "Deaths: final data for 2003," National Vital Statistics Reports, vol. 54, no. 13, pp. 1-120, 2006.

[86] S. Di Donato, "Multisystem manifestations of mitochondrial disorders," Journal of Neurology, vol. 256, no. 5, pp. 693-710, 2009.

[87] J. S. Ingwall, "Energy metabolism in heart failure and remodelling," Cardiovascular Research, vol. 81, no. 3, pp. 412419, 2009.

[88] S. Neubauer, "The failing heart-an engine out of fuel," The New England Journal of Medicine, vol. 356, no. 11, pp. 11401151, 2007.

[89] V. B. Pillai, N. R. Sundaresan, G. Kim et al., "Exogenous NAD blocks cardiac hypertrophic response via activation of the SIRT3-LKB1-AMP-activated kinase pathway," Journal of Biological Chemistry, vol. 285, no. 5, pp. 3133-3144, 2010.

[90] C. P. Hsu, S. Oka, D. Shao, N. Hariharan, and J. Sadoshima, "Nicotinamide phosphoribosyltransferase regulates cell survival through $\mathrm{NAD}^{+}$synthesis in cardiac myocytes," Circulation Research, vol. 105, no. 5, pp. 481-491, 2009.

[91] M. Ruzicka, E. Coletta, R. White, R. Davies, H. Haddad, and F. H.H. Leenen, "Effects of ACE inhibitors on cardiac 
angiotensin II and aldosterone in humans: relevance of lipophilicity and affinity for ACE," American Journal of Hypertension, vol. 23, no. 11, pp. 1179-1182, 2010.

[92] A. Benigni, D. Corna, C. Zoja et al., "Disruption of the Ang II type 1 receptor promotes longevity in mice," Journal of Clinical Investigation, vol. 119, no. 3, pp. 524-530, 2009.

[93] R. Ventura-Clapier, A. Garnier, V. Veksler, and F. Joubert, "Bioenergetics of the failing heart," Biochimica et Biophysica Acta. In press.

[94] R. Ventura-Clapier, A. Garnier, and V. Veksler, "Energy metabolism in heart failure," Journal of Physiology, vol. 555, no. 1, pp. 1-13, 2004.

[95] G. D. Lopaschuk, J. R. Ussher, C. D. L. Folmes, J. S. Jaswal, and W. C. Stanley, "Myocardial fatty acid metabolism in health and disease," Physiological Reviews, vol. 90, no. 1, pp. 207-258, 2010.

[96] G. D. Lopaschuk, D. D. Belke, J. Gamble, T. Itoi, and B. O. Schonekess, "Regulation of fatty acid oxidation in the mammalian heart in health and disease," Biochimica et Biophysica Acta, vol. 1213, no. 3, pp. 263-276, 1994.

[97] S. Neubauer, "The failing heart-an engine out of fuel," The New England Journal of Medicine, vol. 356, no. 11, pp. 11401151, 2007.

[98] M. G. Rosca, E. J. Vazquez, J. Kerner et al., "Cardiac mitochondria in heart failure: decrease in respirasomes and oxidative phosphorylation," Cardiovascular Research, vol. 80, no. 1, pp. 30-39, 2008.

[99] M. F. Allard, B. O. Schonekess, S. L. Henning, D. R. English, and G. D. Lopaschuk, "Contribution of oxidative metabolism and glycolysis to ATP production in hypertrophied hearts," American Journal of Physiology, vol. 267, no. 2, pp. H742H750, 1994.

[100] V. G. Sharov, A. Goussev, M. Lesch, S. Goldstein, and H. N. Sabbah, "Abnormal mitochondrial function in myocardium of dogs with chronic heart failure," Journal of Molecular and Cellular Cardiology, vol. 30, no. 9, pp. 1757-1762, 1998.

[101] H. Taegtmeyer, "Cardiac metabolism as a target for the treatment of heart failure," Circulation, vol. 110, no. 8, pp. 894-896, 2004.

[102] K. P. O. Warburg and E. Negelein, "Ueber den Stoffwechsel der Tumoren," Biochemische Zeitschrift, vol. 152, pp. 319344, 1924.

[103] O. Warburg, "On the origin of cancer cells," Science, vol. 123, no. 3191, pp. 309-314, 1956.

[104] S. Mazurek, C. B. Boschek, F. Hugo, and E. Eigenbrodt, "Pyruvate kinase type M2 and its role in tumor growth and spreading," Seminars in Cancer Biology, vol. 15, no. 4, pp. 300-308, 2005.

[105] H. R. Christofk, M. G. Vander Heiden, M. H. Harris et al., "The M2 splice isoform of pyruvate kinase is important for cancer metabolism and tumour growth," Nature, vol. 452, no. 7184, pp. 230-233, 2008.

[106] M. G. Vander Heiden, J. W. Locasale, K. D. Swanson et al., "Evidence for an alternative glycolytic pathway in rapidly proliferating cells," Science, vol. 329, no. 5998, pp. 1492-1499, 2010.

[107] R. J. DeBerardinis, A. Mancuso, E. Daikhin et al., "Beyond aerobic glycolysis: transformed cells can engage in glutamine metabolism that exceeds the requirement for protein and nucleotide synthesis," Proceedings of the National Academy of Sciences of the United States of America, vol. 104, no. 49, pp. 19345-19350, 2007.

[108] D. R. Wise, R. J. Deberardinis, A. Mancuso et al., "Myc regulates a transcriptional program that stimulates mitochondrial glutaminolysis and leads to glutamine addiction," Proceedings of the National Academy of Sciences of the United States of America, vol. 105, no. 48, pp. 18782-18787, 2008.

[109] R. J. DeBerardinis, J. J. Lum, G. Hatzivassiliou, and C. B. Thompson, "The biology of cancer: metabolic reprogramming fuels cell growth and proliferation," Cell Metabolism, vol. 7, no. 1, pp. 11-20, 2008.

[110] R. Taub, I. Kirsch, C. Morton et al., "Translocation of the c-myc gene into the immunoglobulin heavy chain locus in human Burkitt lymphoma and murine plasmacytoma cells," Proceedings of the National Academy of Sciences of the United States of America, vol. 79, no. 24, pp. 7837-7841, 1982.

[111] M. Schwab, K. Alitalo, K. H. Klempnauer et al., "Amplified DNA with limited homology to myc cellular oncogene is shared by human neuroblastoma cell lines and a neuroblastoma tumour," Nature, vol. 305, no. 5931, pp. 245-248, 1983.

[112] M. M. Nau, B. J. Brooks, J. Battey et al., "L-myc, a new mycrelated gene amplified and expressed in human small cell lung cancer," Nature, vol. 318, no. 6041, pp. 69-73, 1985.

[113] D. E. Jenne, H. Reimann, J.-I. Nezu et al., "Peutz-Jeghers syndrome is caused by mutations in a novel serine threonine kinase," Nature Genetics, vol. 18, no. 1, pp. 38-43, 1998.

[114] J. Zhou, J. Scholes, and J. T. Hsieh, "Characterization of a novel negative regulator (DOC-2/DAB2) of c-Src in normal prostatic epithelium and cancer," Journal of Biological Chemistry, vol. 278, no. 9, pp. 6936-6941, 2003.

[115] C. A. Castaneda, H. Cortes-Funes, H. L. Gomez, and E. M. Ciruelos, "The phosphatidyl inositol 3-kinase/AKT signaling pathway in breast cancer," Cancer and Metastasis Reviews, vol. 29, no. 4, pp. 751-759, 2010.

[116] P. M. Comoglio, M. F. Di Renzo, and G. Gaudino, "Protein tyrosine kinases associated with human malignancies," Annals of the New York Academy of Sciences, vol. 511, pp. 256261, 1987.

[117] I. Hoshino and H. Matsubara, "Recent advances in histone deacetylase targeted cancer therapy," Surgery Today, vol. 40, no. 9, pp. 809-815, 2010.

[118] G. Rose, S. Dato, K. Altomare et al., "Variability of the SIRT3 gene, human silent information regulator Sir2 homologue, and survivorship in the elderly," Experimental Gerontology, vol. 38, no. 10, pp. 1065-1070, 2003.

[119] D. Bellizzi, G. Rose, P. Cavalcante et al., "A novel VNTR enhancer within the SIRT3 gene, a human homologue of SIR2, is associated with survival at oldest ages," Genomics, vol. 85, no. 2, pp. 258-263, 2005.

[120] D. Bellizzi, G. Covello, F. Cianni, Q. Tong, and G. de Benedictis, "Identification of GATA2 and AP-1 activator elements within the enhancer VNTR occurring in intron 5 of the human SIRT3 gene," Molecules and Cells, vol. 28, no. 2, pp. 87-92, 2009.

[121] F. Lescai, H. Blanché, A. Nebel et al., "Human longevity and 11p15.5: a study in 1321 centenarians," European Journal of Human Genetics, vol. 17, no. 11, pp. 1515-1519, 2009. 


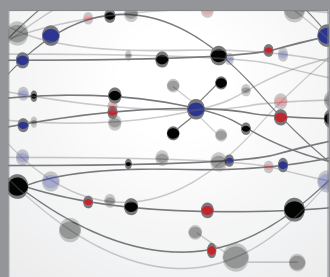

The Scientific World Journal
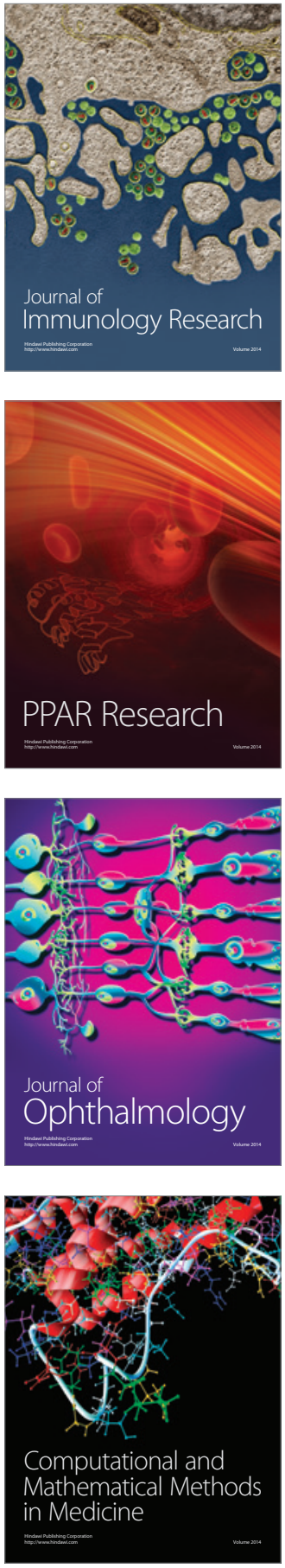

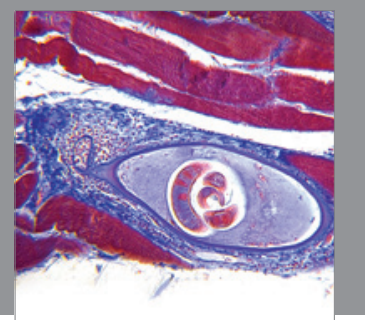

Gastroenterology

Research and Practice
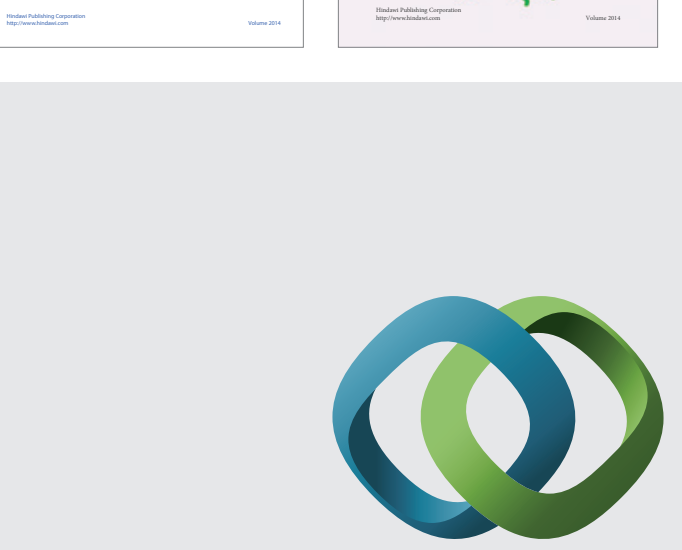

\section{Hindawi}

Submit your manuscripts at

http://www.hindawi.com
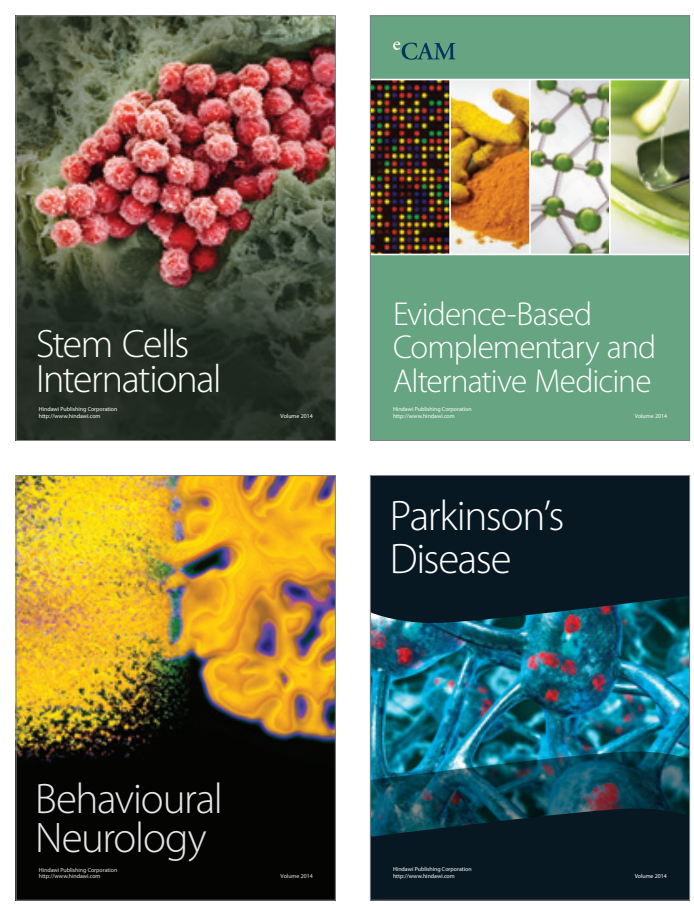

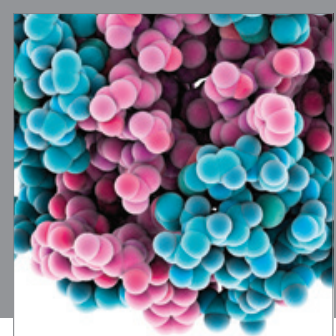

Journal of
Diabetes Research

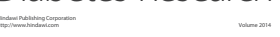

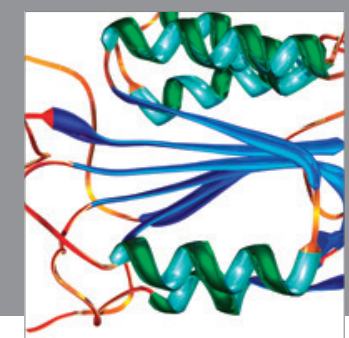

Disease Markers
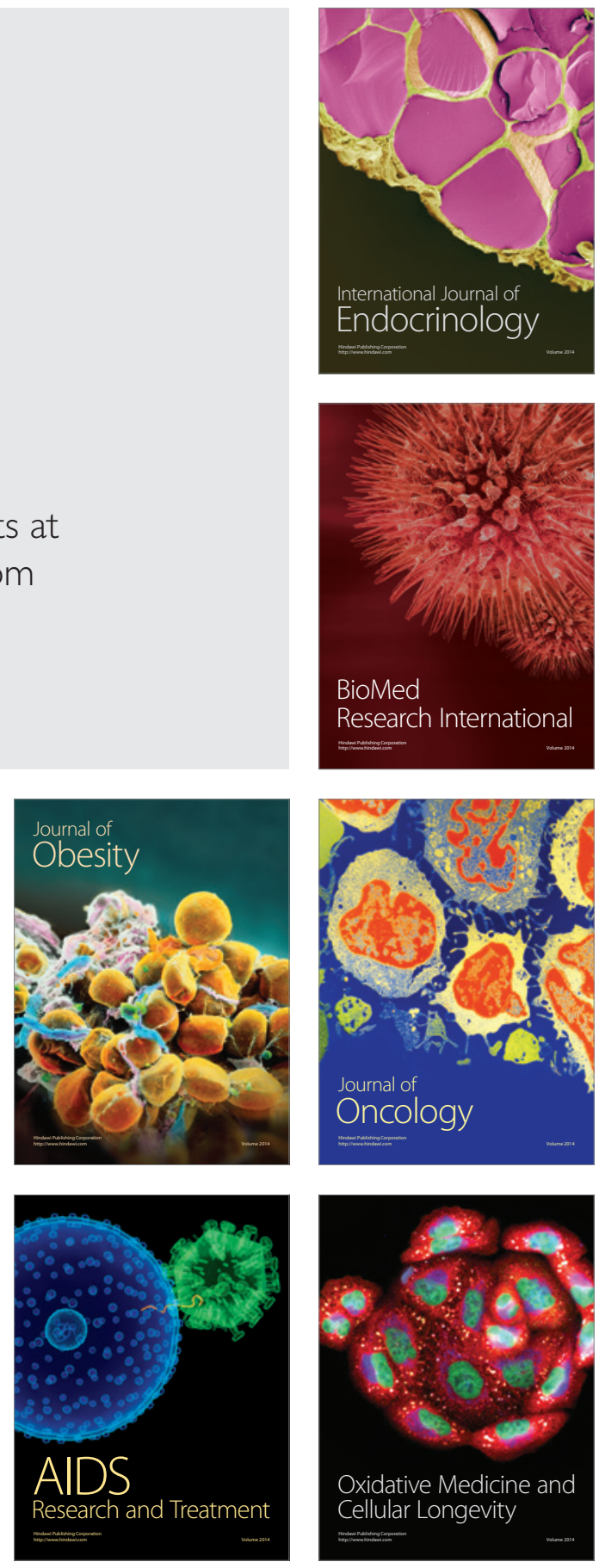